\title{
A Detailed Analysis of the Resonant Frequency and Sensitivity of Flexural Modes of Atomic Force Microscope Cantilevers with a Sidewall Probe Based on a Nonlocal Elasticity Theory
}

\author{
Mohammad Abbasi* - Ardeshir Karami Mohammadi \\ School of Mechanical Engineering, Shahrood University of Technology, Shahrood, Iran
}

\begin{abstract}
In this paper, utilizing a nonlocal elasticity theory, the resonant frequency and sensitivities of an atomic force microscope (AFM) with an assembled cantilever probe (ACP) are studied. This ACP comprises a horizontal cantilever and a vertical extension, and a tip located at the free end of the extension, which makes the AFM capable of topography at the sidewalls of microstructures. First, the governing differential equations of motion and boundary conditions for flexural vibration are obtained via a combination of the basic equations of nonlocal elasticity theory and Hamilton's principle. Afterwards, a closed-form expression for the sensitivity of vibration modes is obtained using the relationship between the resonant frequency and contact stiffness between tip and sample. This analysis provide a better representation of the vibration behaviour of an AFM cantilever with a sidewall probe, for which the effects of the small scale are significant. The results of the nonlocal theory are compared to those of classic beam theory. The evaluation shows that the resonant frequency and sensitivity of the proposed ACP are size dependent, especially when the contact stiffness are high.
\end{abstract}

Keywords: atomic force microscope, assembled cantilever probe, nonlocal elasticity theory, size dependent

\section{INTRODUCTION}

Atomic-force microscopes (AFM) were originally developed to provide high-resolution images of the surface structures of both conductive and insulating samples in both air and liquid environments [1] and [2]. It is also widely used for nano-manipulation and nano-lithography in micro/nano-electromechanical systems (MEMS/NEMS) [3] and [4]. The contact between the tip of the elastic cantilever and the sample induces a dynamic interaction. The imaging rate and contrast of topographical images significantly depend on the resonant frequency and sensitivity of the AFM cantilever. Hence, investigations of the dynamic behaviour of the AFM microcantilever seem to be crucial. To date, many researchers have studied the dynamic response of the AFM cantilever [5] to [8].

Conventional AFMs consist of a microcantilever with a sharp conical or pyramidal tip located at its free end and play an important role in nano-scale surface measurements. Unfortunately, their probe tips never come in close proximity to sidewalls, regardless of how sharp and thin the tips are. Therefore, an urgent need for nano-scale surface measurements at sidewalls exists. In order to overcome the limitations of conventional AFMs, Dai et al. [9] proposed assembled cantilever probes (ACPs) for direct and non-destructive sidewall measurement of nano- and microstructures. Utilizing classic beam theory, Chang et al. [10] analysed the sensitivity and the resonant frequency an assembled cantilever probe, consisting of a horizontal AFM cantilever and a vertical extension. Kahrobaiyan et al. [11] studied the resonant frequencies and flexural sensitivities of another form of ACPs proposed by Dai et al. [12], comprising a horizontal cantilever, a vertical extension and two tips located at the free ends of the cantilever and the extension.

Beams used in MEMS and NEMS, such as AFMs, have thicknesses in the order of microns and sub-microns. The size dependency of static and dynamic behaviour of the micro-scale structures have been verified in the experimental observations [13] and [14]. For example, McFarland and Colton [15] detected a considerable difference between the stiffness values predicted by the classic beam theory and the stiffness values obtained during bending tests of polypropylene microcantilevers. In the microtorsion test of thin copper wires, Fleck et al. [16] observed that a decrease in wire diameters from 170 to 12 results in a significant increase of the torsional hardening. Lam et al. [17] observed a significant enhancement of bending rigidity caused by the beam thickness reduction in microbending tests of beams made of epoxy polymers. The lack of an internal material length scale parameter in the classic continuum models causes some deficiencies in studying small scale nanostructures. Hence, sizedependent continuum mechanics models such as the strain gradient theory [18] and [19], couple stress theory [20] and [21], modified couple stress theory [22] and nonlocal elasticity theory [23] and [24] are used. Nonlocal elasticity theory, which assumes that the stress state at a given point to be dependent on the strain states at all points in the body, have been the 
subject of much attention in nano-mechanics because of its efficiency.

The nonlocal theory of elasticity has been used to investigate many fields, such as the lattice dispersion of elastic waves, wave propagation in composites, fracture mechanics, dynamic and the static analysis of carbon nanotubes and nano-rods, surface tension fluids, etc. [25] and [26]. Using beam-and-shell theories, Wang et al. [27] applied the nonlocal elasticity constitutive equations to study the vibration and buckling of carbon nanotubes. Using the nonlocal constitutive relations of Eringen [23], Reddy [28] reformulated the equations of motion of various beam theories, including the Euler-Bernoulli, Timoshenko, higher order, and Levinson beam models, using different nonlocal beam theories for bending, buckling and free vibration problems. A generalized nonlocal beam theory to derive the governing equations for different beam theories was proposed by Aydogdu [29]. Next, the effects of nonlocality and length of beams were investigated in some detail for each considered problem. Thai [30] proposed a nonlocal shear deformation beam theory for the bending, buckling, and vibration of nano-beams based on the nonlocal constitutive relations of Eringen [23]. His theory accounted for a quadratic variation of the shear strains across the thickness, and satisfied the zerotraction boundary conditions on the top and bottom surfaces of the beam without using shear correction factor. Utilizing this theory, Gheshlaghi and Mirzaei [31] studied the frequency shift of microcantileverbased sensors. They found that the normalized natural frequencies obtained for the nonlocal cantilever microbeam become smaller than those for its local counterpart .

Civalek and Demir [32] developed an elastic nonlocal beam model for the bending analysis of microtubules (MTs) based on the Euler-Bernoulli beam theory. They concluded that the nonlocal continuum theory approach is superior to average (local) elasticity, especially for some boundary conditions

Since ACPs' cantilevers have thicknesses in the order of microns and sub-microns, studying their dynamic behaviour utilizing a non-classic beam theory such as nonlocal beam theory seems necessary.

In this study, utilizing nonlocal elasticity theory, the sensitivity and resonant frequency of flexural vibration modes of an ACP proposed by Dai et al.[9], consisting of a horizontal cantilever and a vertical extension, are analysed.

\section{THE NONLOCAL ELASTICITY THEORY}

According to the nonlocal elasticity theory proposed by Eringen [23] and [24], the stress components at a reference point $x$ in an elastic continuum depend not only on the strain components at the same position $x$ but also on all other points of the body. Hence, the well-known nonlocal constitutive relation in terms of a partial differential form is expressed as [23]:

$$
\left(1-\mu \nabla^{2}\right) \sigma_{i j}=C_{i j m n} \varepsilon_{m n}, \quad \mu=e_{0}^{2} a^{2},
$$

where $\sigma_{i j}$ is the nonlocal stress tensor components at point $x, c_{i j m n}$ is the fourth-order elasticity tensor, $\varepsilon_{m n}$ is the strain tensor, $\mu$ is the nonlocal parameter, and $e_{0}$ and $a$ are the material constant and internal characteristic length, respectively, which incorporate the small-scale effect. The nonlocal parameter depends on the boundary conditions, chirality, mode shapes and type of motion [33].

Assuming that the nonlocal behaviour is negligible in the thickness direction, the nonlocal constitutive relation in Eq. (1) takes the following special relation for beams [34].

$$
\sigma_{x x}-\mu \frac{\partial^{2} \sigma_{x x}}{\partial x^{2}}=E \varepsilon_{x x},
$$

where $E$ is Young's modulus. It should be noted that for the case of $\mu=0$, we obtain the constitutive relations of local beam theories.

\section{ANALYSIS OF THE DYNAMIC BEHAVIOUR OF ACP MICROCANTILEVERS}

The proposed type of AFM ACP developed in this study comprises a horizontal cantilever, a vertical extension and a tip located at the free end of the extension, which makes the AFM capable of topography at the sidewalls of microstructures. The geometrical parameters and configuration of this ACP are depicted in Fig. 1. The horizontal cantilever and vertical extension have a uniform cross section thickness $b$, width $w$, whose lengths are $L$ and $H$, respectively, and the small length tip is $s$. Considering the ratio of the extension rigidity to cantilever rigidity, the deflection of the extension in comparison with the cantilever deflection can be neglected, and it can be assumed that the extension is rigid. Therefore, the cantilever of ACP experiences flexural vibrations during contact with the sample. The interaction between AFM tip and microstructure sidewall results in torsional vibration in the AFM probe. As shown in Fig. 1, the ACP interacts with the sample surface 
at its tip by normal springs $K_{n}$ for normal interaction and lateral springs $K_{l}$ for lateral interaction. $x$ is the coordinate along the centre of the cantilever, while $w(x, t)$ is the vertical deflection in $x$-direction at time $t$.

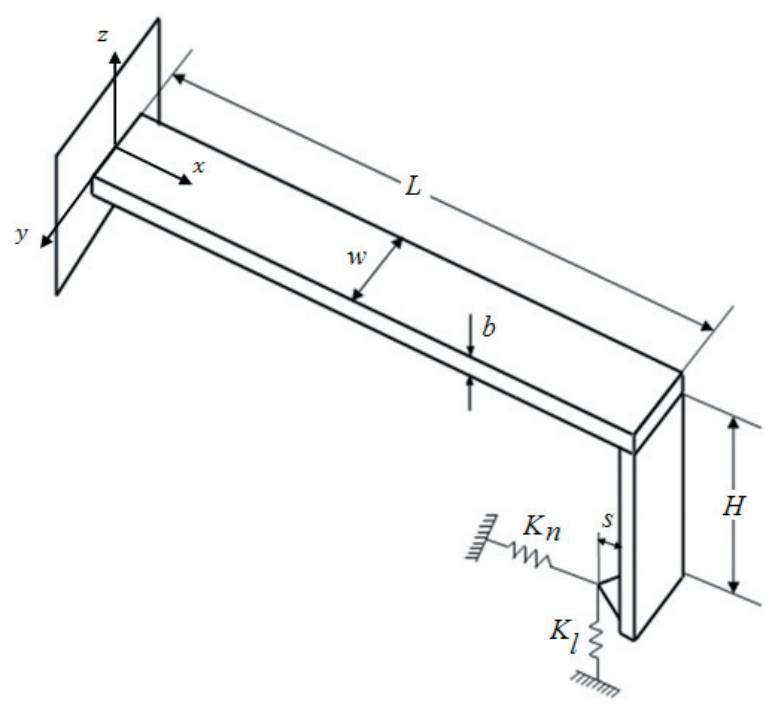

Fig. 1. Schematic diagram of an AFM cantilever micro-assembled with vertical extension and a tip located at the free end of the vertical extension for flexural vibration

Based on the flexural vibration of the ACP microcantilever depicted in Fig. 1, the components of displacement vector $u$ are expressed as:

$$
u_{x}=-z \frac{\partial w(x, t)}{\partial x}, \quad u_{y}=0, \quad u_{z}=w(x, t),
$$

where $u_{x}, u_{y}$ and $u_{z}$ denote the displacement along $x, y$ and $z$ axes, respectively.

The only non-zero strains of the Euler-Bernoulli beam theory are:

$$
\varepsilon_{x x}=-z \frac{\partial^{2} w(x, t)}{\partial x^{2}} .
$$

Considering Eqs. (2) and (4) and using the moment strain relation:

$$
M=\int_{A} z \sigma_{x x} d A
$$

one obtains:

$$
M-\mu \frac{\partial^{2} M}{\partial x^{2}}=-E I \frac{\partial^{2} w(x, t)}{\partial x^{2}},
$$

where $I$ denotes the second moment of area about the $y$-axis. Then the strain energy $U$ in a deformed isotropic linear elastic material occupying region $\Omega$ is given by:

$$
U=\frac{1}{2} \int_{\Omega} \sigma_{i j} \varepsilon_{i j} d v,
$$

by substituting Eq. (4) into Eq. (7) and also considering Eq. (5), one obtains the following expression for the strain energy $U$ :

$$
U=-\int_{0}^{L} M \frac{\partial^{2} w(x, t)}{\partial x^{2}} d x
$$

Considering the proposed ACP microcantilever depicted in Fig. 1 yields the following result for the elastic potential energy of the system:

$$
\begin{aligned}
U & =-\int_{0}^{L} M \frac{\partial^{2} w(x, t)}{\partial x^{2}} d x+\frac{1}{2} K_{n}\left(H \frac{\partial w(L, t)}{\partial x}\right)^{2}+ \\
& +\frac{1}{2} K_{l}\left(w(x, t)-s \frac{\partial w(x, t)}{\partial x}\right)^{2} .
\end{aligned}
$$

Denoting the mass per unit length of cantilever and extension by $\rho$ and $\rho_{e}$, respectively, and the extension mass and mass moment of inertia by $M_{e}$ and $J_{e}$, and also the cantilever and extension areas by $A$ and the mass moment of inertia of the extension by $J_{h}$, the kinetic energy of the system due to the velocity of its particles along the $z$-direction can be written as:

$$
\begin{aligned}
T & =\frac{1}{2} \int_{0}^{L} \rho A\left(\frac{\partial w(x, t)}{\partial t}\right)^{2} d x+\frac{1}{2} M_{e}\left(\frac{\partial w(L, t)}{\partial t}\right)^{2}+ \\
& +\frac{1}{2} J_{e}\left(\frac{\partial w(L, t)}{\partial x \partial t}\right)^{2},
\end{aligned}
$$

where $M_{e}=\rho_{e} A H$ and $J_{e}=\frac{1}{3} M_{e} H^{2}=\frac{1}{3} \rho_{e} A H^{3}$.

Bearing in mind the aforementioned expressions for $U$ and $T$, the dynamic governing equation of this ACP microcantilever as well as boundary conditions can be determined with the aid of Hamilton's principle:

$$
\int_{t_{1}}^{t_{2}}\left(\delta T-\delta U+\delta W_{e}\right) d t=0
$$

in the above equation, $W_{e}$ is the work done by external classic and higher-order torques which are assumed to be zero. Inserting Eqs. (9) and (10) into Eq. (11) then leads to:

$$
\begin{gathered}
\frac{\partial^{2} M(x, t)}{\partial x^{2}}-\rho A \frac{\partial^{2} w(x, t)}{\partial x^{2}}=0, \\
w(0, t)=0, \quad \frac{\partial w(0, t)}{\partial x}=0,
\end{gathered}
$$




$$
\begin{gathered}
M_{e} \frac{\partial^{2} w(L, t)}{\partial t^{2}}+\left.\frac{\partial M}{\partial x}\right|_{x=L}+K_{l} w(L, t)=0, \\
-J_{e} \frac{\partial^{3} w(L, t)}{\partial x \partial t^{2}}+M-K_{n} H^{2} \frac{\partial w(L, t)}{\partial x}+ \\
+K_{l} s w(L, t)=0
\end{gathered}
$$

by substituting Eq. (12) into Eq. (6), one obtains the following expression for $M$ :

$$
M=-E I \frac{\partial^{2} w(x, t)}{\partial x^{2}}+\mu \rho A \frac{\partial^{2} w(x, t)}{\partial t^{2}} .
$$

Inserting Eq. (16) into Eqs. (12) to (15), the dynamic governing equation and the boundary conditions can be rewritten as:

$$
\begin{gathered}
E I \frac{\partial^{4} w(x, t)}{\partial x^{4}}+\rho A \frac{\partial^{2} w(x, t)}{\partial t^{2}}-\mu \rho A \frac{\partial^{4} w(x, t)}{\partial t^{2} \partial x^{2}}=0 \\
w(0, t)=0, \quad \frac{\partial w(0, t)}{\partial x}=0 \\
\mu \rho A \frac{\partial^{3} w(L, t)}{\partial t^{2} \partial x}-E I \frac{\partial^{3} w(L, t)}{\partial x^{3}}+ \\
+M_{e} \frac{\partial^{2} w(L, t)}{\partial t^{2}}+K_{l} w(L, t)=0 \\
E I \frac{\partial^{2} w(L, t)}{\partial x^{2}}-\mu \rho A \frac{\partial^{2} w(L, t)}{\partial t^{2}}+J_{e} \frac{\partial^{3} w(L, t)}{\partial x \partial t^{2}}+ \\
+K_{n} H^{2} \frac{\partial w(L, t)}{\partial x}-K_{l} s w(L, t)=0 .
\end{gathered}
$$

The dimensionless variables are defined as:

$$
\begin{aligned}
& X=\frac{x}{L}, \quad W=\frac{w}{L}, \quad \tau=\frac{t}{L^{2}} \sqrt{\frac{E I}{\rho A}}, \\
& S=\frac{s}{L}, \quad m=\frac{\rho_{e}}{\rho}, \quad e=\frac{\mu}{L^{2}}, \\
& h=\frac{H}{L}, \quad \beta_{n}=\frac{K_{n} L^{3}}{E I}, \quad \beta_{l}=\frac{K_{l} L^{3}}{E I} .
\end{aligned}
$$

Applying this set of dimensionless variables quantities given in Eq. (21) into Eqs. (17) to (20) yields the normalized form of free vibration equation and the associated boundary conditions as:

$$
\begin{gathered}
\frac{\partial^{4} W(X, \tau)}{\partial X^{4}}+\frac{\partial^{2} W(X, \tau)}{\partial \tau^{2}}-e \frac{\partial^{4} W(X, \tau)}{\partial \tau^{2} \partial X^{2}}=0 \\
W(0, \tau)=0, \quad \frac{\partial W(0, \tau)}{\partial X}=0
\end{gathered}
$$

$$
\begin{gathered}
e \frac{\partial^{3} W(1, \tau)}{\partial \tau^{2} \partial X}-\frac{\partial^{3} W(1, \tau)}{\partial X^{3}}+m h \frac{\partial^{2} W(1, \tau)}{\partial \tau^{2}}+ \\
+\beta_{l} W(1, \tau)=0, \\
\frac{\partial^{2} W(1, \tau)}{\partial X^{2}}-e \frac{\partial^{2} W(1, \tau)}{\partial \tau^{2}}+\frac{1}{3} m h^{3} \frac{\partial^{3} W(1, \tau)}{\partial X \partial \tau^{2}}+ \\
+\beta_{n} h^{2} \frac{\partial W(1, \tau)}{\partial X}-\beta_{l} S W(1, \tau)=0 .
\end{gathered}
$$

Assuming a harmonic solution for $Y$ as $W(X, \tau)=V(X) e^{i \omega \tau}$ results in the following ordinary differential equation and boundary conditions:

$$
\begin{gathered}
\frac{d^{4} V(X)}{\partial X^{4}}+e \omega^{2} \frac{d^{2} V(X)}{\partial X^{2}}-\omega^{2} V(X)=0, \\
V(0)=\frac{d V(0)}{d X}=0 \\
e \omega^{2} \frac{d V(1)}{d X}+\frac{d^{3} V(1)}{d X^{3}}+r_{1} V(1)=0, \\
\frac{d^{2} V(1)}{d X^{2}}+r_{2} \frac{d V(1)}{d X}+r_{3} V(1)=0
\end{gathered}
$$

where the angular frequency is denoted by $\omega$, and $r_{1}$, $r_{2}$ and $r_{3}$ are defined as:

$$
\begin{aligned}
& r_{1}=m h \omega^{2}-\beta_{l}, \\
& r_{2}=\beta_{n} h^{2}-\frac{1}{3} m h^{3} \omega^{2}, \\
& r_{3}=e \omega^{2}-\beta_{l} S .
\end{aligned}
$$

The solution of Eq. (26) is written as:

$$
\begin{aligned}
V(X) & =a_{1} \sinh \lambda_{1} X+a_{2} \cosh \lambda_{1} X+ \\
& +a_{3} \sin \lambda_{2} X+a_{4} \cos \lambda_{2} X,
\end{aligned}
$$

where $a_{i}(i=1, \ldots, 4)$ are some constants and $\lambda_{1}$ and $\lambda_{2}$ are defined as:

$$
\begin{aligned}
& \lambda_{1}=\sqrt{\frac{-e \omega^{2}+\sqrt{(e \omega)^{2}+4 \omega^{2}}}{2}}, \\
& \lambda_{2}=\sqrt{\frac{e \omega^{2}+\sqrt{(e \omega)^{2}+4 \omega^{2}}}{2}} .
\end{aligned}
$$

Applying boundary conditions (27) to (29) into Eq. (30), the characteristics equation can be found:

$$
C(\omega, \beta)=U_{1} U_{4}-U_{2} U_{3}=0,
$$

where 


$$
\begin{gathered}
U_{1}=e \omega^{2} \lambda_{2}\left(-\cosh \lambda_{1}+\cos \lambda_{2}\right)-\lambda_{1}^{2} \lambda_{2} \cosh \lambda_{1}- \\
-\lambda_{2}^{3} \cos \lambda_{2}+r_{1}\left(\frac{-\lambda_{2}}{\lambda_{1}} \sinh \lambda_{1}+\sin \lambda_{2}\right) \\
U_{2}=-e \omega^{2}\left(\lambda_{1} \sinh \lambda_{1}+\lambda_{2} \sin \lambda_{2}\right)+ \\
+\left(-\lambda_{1}^{3} \sinh \lambda_{1}+\lambda_{2}^{3} \sin \lambda_{2}\right)+r_{1}\left(-\cosh \lambda_{1}+\cos \lambda_{2}\right), \\
U_{3}=-\lambda_{1} \lambda_{2} \sinh \lambda_{1}-\lambda_{2}^{2} \sin \lambda_{2}+ \\
+r_{2} \lambda_{2}\left(-\cosh \lambda_{1}+\cos \lambda_{2}\right)+r_{3}\left(\frac{-\lambda_{2}}{\lambda_{1}} \sinh \lambda_{1}+\sin \lambda_{2}\right) \\
U_{4}=-\lambda_{1}^{2} \cosh \lambda_{1}-\lambda_{2}^{2} \cos \lambda_{2}- \\
-r_{2}\left(\lambda_{1} \sinh \lambda_{1}+\lambda_{2} \sin \lambda_{2}\right)+r_{3}\left(-\cosh \lambda_{1}+\cos \lambda_{2}\right)
\end{gathered}
$$

The dimensionless sensitivity of an AFM ACP, $S$ is defined as the differentiation of the dimensionless natural frequency with respect to the dimensionless surface contact stiffness, i.e.:

$$
S=\frac{\partial \omega}{\partial \beta_{l}}=-\left(\frac{\partial C}{\partial \beta_{l}}\right) /\left(\frac{\partial C}{\partial \omega}\right) .
$$

\section{RESULTS AND DISCUSSION}

This section presents the results of the vibration analysis of an AFM ACP based on the nonlocal elasticity theory. The analytical expressions were obtained for the sensitivity and resonant frequency of flexural modes to indicate a better representation of the flexural behaviour of an AFM with sidewall probe where the small-scale effect are significant. To this end, we consider the geometric and material parameters of the cantilever and extension to be $\rho=2330 \mathrm{~kg} / \mathrm{m}^{3}, \rho_{e}=3440 \mathrm{~kg} / \mathrm{m}^{3}$ and $h=0.5$. The default value for the normalized nonlocal parameter, $e$ is considered to be $e=0.03$ and also the lateral and normal contact stiffness are assumed as $\beta=0.9 \beta_{l}$ [10], [11] and [30].

The dimensionless flexural resonant frequency of the first and second modes of the AFM cantilever based on nonlocal elasticity and classic beam theories are compared in Figs. 2 and 3 for different values of normalized nonlocal parameter. It should be noted that for the case $e=0$, nonlocal elasticity theory is reduced to classic beam theory. Considering these two figures, it can be found that the resonant frequency takes at a low constant value for lower values of contact stiffness. However, by increasing the contact stiffness, the resonant frequency rises dramatically

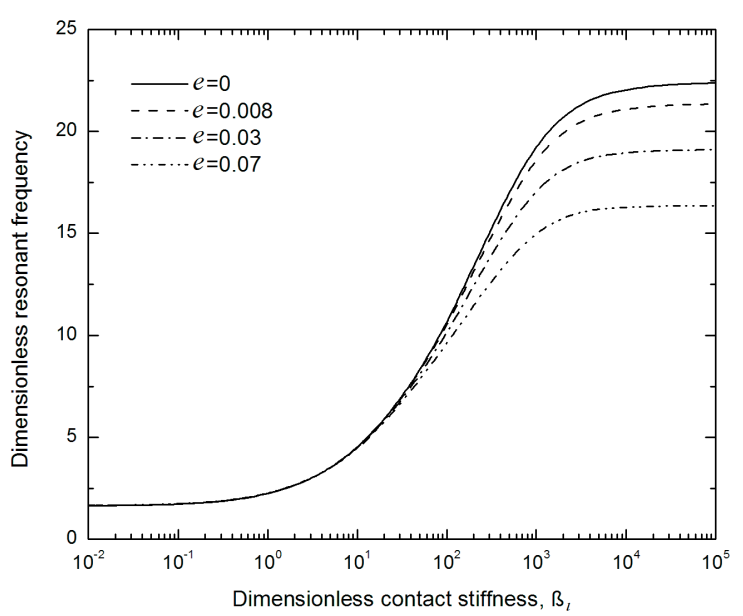

Fig. 2. The first dimensionless flexural resonant frequency for an AFM cantilever at various values of normalized nonlocal parameter

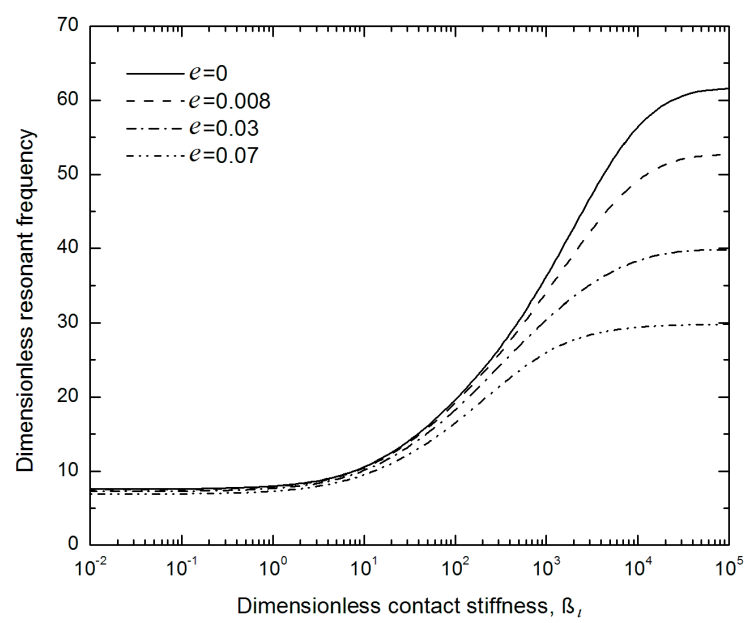

Fig. 3. The second dimensionless flexural resonant frequency for an AFM cantilever at various values of normalized nonlocal parameter

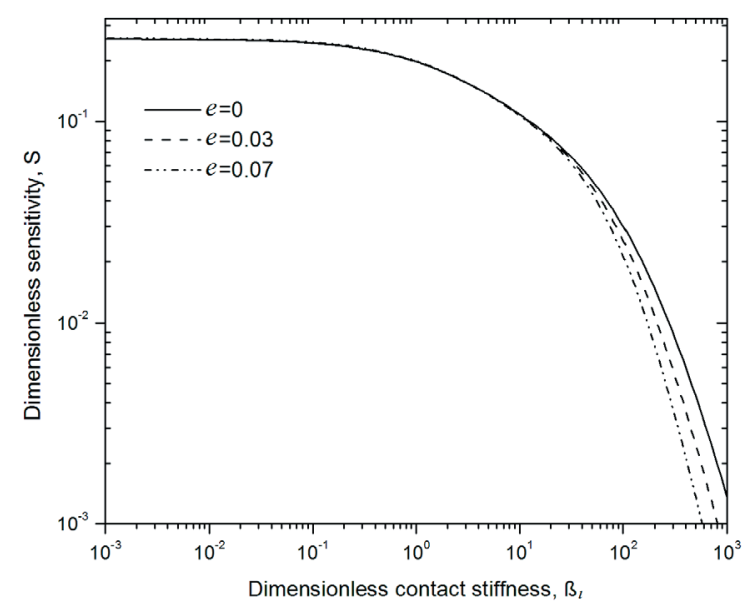

Fig. 4. The first dimensionless flexural sensitivity for an AFM cantilever at various values of normalized nonlocal parameter 
until reaches another constant value at very high value of contact stiffness. These figures also indicate that for large values of contact stiffness, the resonant frequencies obtained by nonlocal theory are smaller than those calculated by classic beam theory. At these values of contact stiffness, an increase in normalized nonlocal parameter decreases the resonant frequency. Furthermore, it can be inferred that the resonant frequency of the second mode is more sensitive to the change in the normalized nonlocal parameter.

Figs. 4 and 5 illustrate the change in the first and second flexural sensitivity modes due to the change in contact stiffness, $\beta_{l}$ and normalized nonlocal parameter, $e$. According to these figures, the sensitivity is maximum when the contact stiffness is low. In the first mode, an increase in contact stiffness decreases the sensitivity of the first mode, while a similar phenomenon happens in the second mode after the sensitivity experiences a minimum around the point $\beta_{l}=10$ and a peak approximately at the point $\beta_{l}=10^{3}$, respectively. Furthermore, for the higher values of contact stiffness, the sensitivities of the first mode predicted by nonlocal theory is smaller than those obtained by classic beam theory. There is a similar situation in the second mode in all ranges of contact stiffness.

The flexural resonant frequency of the first mode due to the change in dimensionless contact stiffness, $\beta_{l}$, and vertical extension length, $h$, is depicted in Fig. 6 . It can be seen that the resonant frequency increases as the contact stiffness grows but reduces as the vertical extension enlarges.

The first flexural sensitivity of the sidewall tip versus dimensionless contact stiffness, $\beta_{l}$ and various vertical extension length, $h$ is shown in Fig. 7. From this figure, it can be concluded that an increase in vertical extension length reduces the flexural sensitivity when the contact stiffness is low. The situation is reversed for very high values of contact stiffness $\left(\beta_{l}>100\right)$.

Fig. 8 represents the first flexural sensitivity of mode 1 as a function of vertical extension length, and $h$ at different values of normalized nonlocal parameter for $\beta_{l}=10^{3}$. It should be noted from Fig. 4 that the sensitivities of first mode differ from each other for different values of nonlocal parameters when the contact stiffness is high. Hence, the contact stiffness is assumed as $\beta_{l}=10^{3}$ in Fig. 8. From this figure, it can be inferred that the effect of change in vertical extension length $h$ on the flexural sensitivity predicted by classic beam theory $(e=0)$ is significant; and a rise in the dimension length of vertical extension $h$ increases the sensitivity dramatically. For the sensitivity calculated

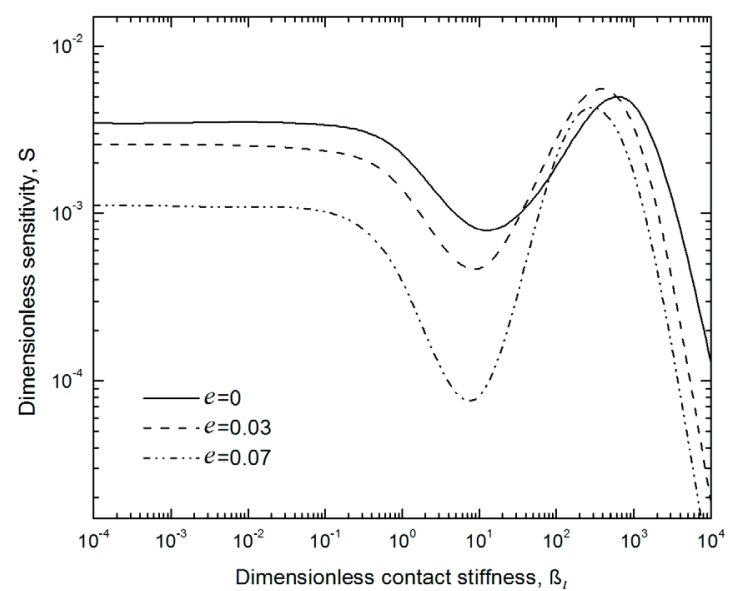

Fig. 5. The second dimensionless flexural sensitivity for an AFM cantilever at various values of normalized nonlocal parameter

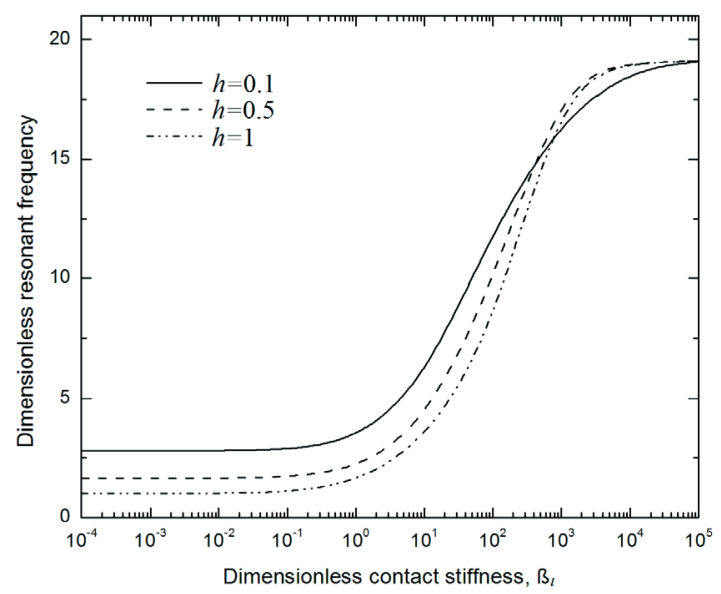

Fig. 6. The first dimensionless flexural resonant frequency for an AFM cantilever at various values of dimensionless length of the vertical extension

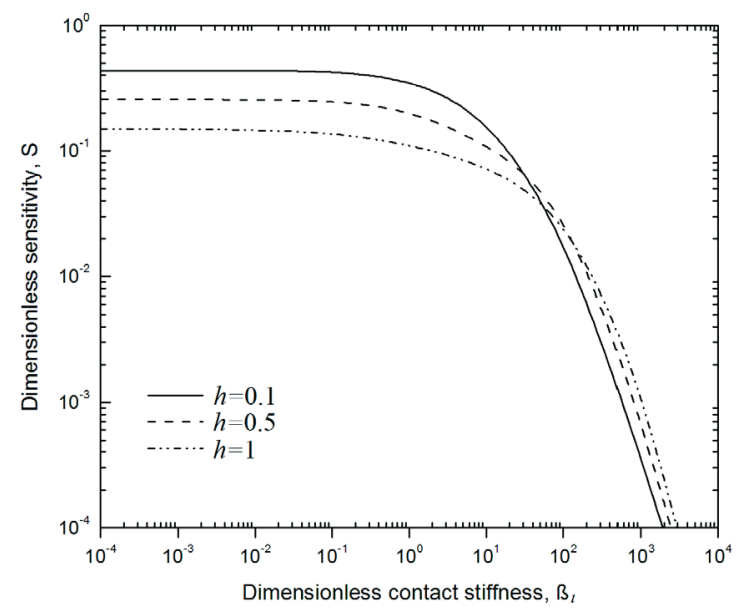

Fig. 7. The first dimensionless flexural sensitivity for an AFM cantilever at various values of dimensionless length of the vertical extension 


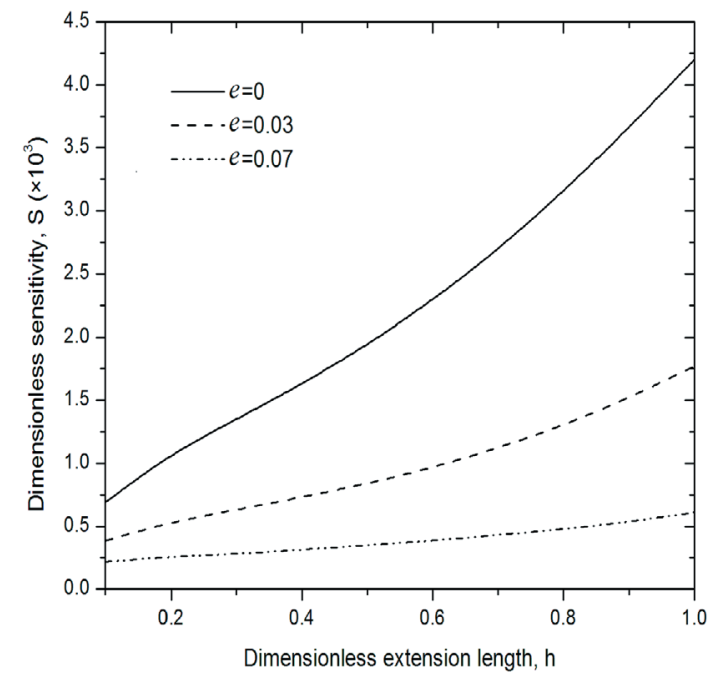

Fig. 8. The first dimensionless flexural sensitivity for an AFM cantilever as a function of dimensionless length of the vertical extension at various normalized nonlocal parameter

by nonlocal elasticity theory, an increase in the normalized nonlocal parameter reduces the effect of vertical extension length on the sensitivity.

\section{CONCLUSION}

In this study, the equations of motion and boundary conditions for flexural vibration of an AFM cantilever with sidewall probe have been derived utilizing nonlocal elasticity theory; analytical solutions are then presented to bring out the effect of nonlocal parameters on the resonant frequency and flexural sensitivity of the first two modes. According to the results, the sensitivities and resonant frequencies predicted by nonlocal elasticity theory are smaller than those evaluated by classic beam theory. It can also be determined that the difference between either the resonant frequency or the flexural sensitivity obtained by nonlocal elasticity theory and those calculated by classic beam theory are more significant for the second mode. Moreover, an increase in the vertical extension length diminishes the resonant frequency, while reducing the sensitivity for low values of contact stiffness and increases it for high values of contact stiffness. Furthermore, a rise in the normalized nonlocal parameter decreases the effect of vertical extension length on the sensitivity.

\section{REFERENCES}

[1] Mazeran, P.E., Loubet, J.L. (1999). Normal and lateral modulation with a scanning force microscope, an analysis: implication in quantitative elastic and friction imaging. Tribology Letters, vol. 7, p. 199-212, DOI:10.1023/A:1019142025011.

[2] Garcia, R., Perez, R. (2002). Dynamic atomic force microscopy methods. Surface Science Reports, vol. 47, no. 6-8, p. 197-301, DOI:10.1016/S01675729(02)00077-8.

[3] Davis, Z.J., Abadal, G., Hansen, O., Borise, X., Barniol, N., Murano, P.F., Boisen, A. (2003). AFM lithography of aluminum for fabrication of nanomechanical systems. Ultramicroscopy, vol. 97, no. 1-4, p. 467-472, DOI:10.1016/S0304-3991(03)00075-5.

[4] Chang, W.J., Fang, T.H., Weng, C.I. (2004). Inverse determination of the cutting force on nanoscale processing using atomic force microscopy. Nanotechnology, vol. 15, no. 5, p. 427-430, DOI:10.1088/0957-4484/15/5/004.

[5] Mahdavi, M.H., Farshidianfar, A., Tahani, M., Mahdavib, S., Dalirc, H. (2008). A more comprehensive modeling of atomic force microscope cantilever. Ultramicroscopy, vol. 109, no. 1, p. 54-60, DOI:10.1016/j.ultramic.2008.08.003.

[6] Wu, T.-S., Chang, W.-J., Hsu, J.-C. (2004). Effect of tip length and normal and lateral contact stiffness on the flexural vibration responses of atomic force microscope cantilevers. Microelectronic Engineering, vol. 71, no. 1, p. 15-20, DOI:10.1016/j.mee.2003.08.009.

[7] Abbasi, M., Mohammadi, A.K. (2010). A new model for investigating the flexural vibration of an atomic force microscope cantilever. Ultramicroscopy, vol. 110, no. 11, p.1374-1379, DOI:10.1016/j.ultramic.2010.07.005.

[8] Lee, H.-L., Chang, W.-J. (2011) Sensitivity of $\mathrm{V}$-shaped atomic force microscope cantilevers based on a modified couple stress theory. Microelectronic Engineering, vol. 88, no 11, p. 3214-3218, DOI:10.1016/j.mee.2011.09.001.

[9] Dai, G., Wolff, H., Pohlenz, F, Danzebrink, H.U., Wilkening, G. (2006). Atomic force probe for sidewall scanning of nano- and microstructures. Applied Physics Letters, vol. 88, no. 17, 171908, DOI:10.1063/1.2198516.

[10] Chang, W.J., Lee, H.L., Chen, T.Y. (2008). Study of the sensitivity of the first four flexural modes of an AFM cantilever with a sidewall probe. Ultramicroscopy, vol. 108, no.7, p.619-624, DOI:10.1016/j. ultramic.2007.10.003.

[11] Kahrobaiyan, M.H., Ahmadian, M.T., Haghighi, P., Haghighi, A. (2010). Sensitivity and resonant frequency of an AFM with sidewall and top-surface probes for both flexural and torsional modes. International Journal of Mechanical Sciences, vol. 52, no. 1, p. 13571365, DOI:10.1016/j.ijmecsci.2010.06.013.

[12] Dai, G., Wolff, H., Weimann, T., Xu, M., Pohlenz, F., Danzebrink, H.-U. (2007). Nanoscale surface measurements at sidewalls of nano- and microstructures. Measurement Science and Technology, vol. 18, no. 2, p. 334-341, DOI:10.1088/0957-0233/18/2/ S03. 
[13] Stolken, J.S., Evans, A.G. (1998). Microbend test method for measuring the plasticity length scale. Acta Materialia, vol. 46, no. 1, p. 5109-5115, DOI:10.1016/ S1359-6454(98)00153-0.

[14] Chong, A.C.M., Lam, D.C.C. (1999). Strain gradient plasticity effect in indentation hardness of polymers. Journal of Materials Research, vol. 14, no. 10, p. 41034110, DOI:10.1557/JMR.1999.0554.

[15] McFarland, A.W., Colton, J.S. (2005). Role of material microstructure in plate stiffness with relevance to microcantilever sensors. Journal of Micromechanics and Microengineering, vol. 15, no. 5, p. 1060-1067, DOI:10.1088/0960-1317/15/5/024.

[16] Fleck, N.A., Muller, G.M., Ashby, M.F. Hutchinson, J.W. (1994). Strain gradient plasticity: theory and experiment. Acta Metallurgica et Materialia, vol. 42, no. 2, p. 475-487, DOI:10.1016/0956-7151(94)905029.

[17] Lam, D.C.C., Yang, F., Chong, A.C.M., Wanga, J., Tonga, P. (2003). Experiments and theory in strain gradient elasticity. Journal of the Mechanics and Physics of Solids, vol. 51, no. 8, p. 1477-1508, DOI:10.1016/S0022-5096(03)00053-X.

[18] Fleck, N.A., Hutchinson, J.W. (1997) Strain Gradient Plasticity. Academic Press, New York.

[19] Kong, S., Zhou, S., Nie, Z., Wang, K. (2009). Static and dynamic analysis of micro beams based on strain gradient elasticity theory. International Journal of Engineering Science, vol. 47, no. 4, p. 487-498, DOI:10.1016/j.ijengsci.2008.08.008.

[20] Toupin, R.A. (1962). Elastic materials with couple stresses. Archive for Rational Mechanics and Analysis, vol. 11, no. 1, p. 385-414, DOI:10.1007/BF00253945.

[21] Mindlin, R.D., Tiersten, H.F. (1962). Effects of couplestresses in linear elasticity. Archive for Rational Mechanics and Analysis, vol. 11, no. 1, p. 415-448, DOI:10.1007/BF00253946.

[22] Kong, S, Zhou, S, Nie, Z, Wang, K. (2008). The size-dependent natural frequency of Bernoulli-Euler micro beams. International Journal of Engineering Science, vol. 46, no. 5, p. 427-437, DOI:10.1016/j. ijengsci.2007.10.002.

[23] Eringen, A.C. (1972), Nonlocal polar elastic continua. International Journal of Engineering Science, vol. 10, no. 1, p. 1-16, DOI:10.1016/0020-7225(72)90070-5.

[24] Eringen, A.C. (1983). On differential equations of nonlocal elasticity and solutions of screw dislocation and surface waves. Journal of Applied Physics, vol. 54, no. 9, p. 4703-4710, DOI:10.1063/1.332803.

[25] Wang, Q. (2005). Wave propagation in carbon nanotubes via nonlocal continuum mechanics. Journal of Applied Physics, vol. 98, no. 12, p. 124301-1243016: 124301.

[26] Xu, M. (2006). Free transverse vibrations of nano-tomicron scale beams. Proceedings of the Royal Society $A$, vol. 462, no. 2074, p. 2977-2995, DOI:10.1098/ rspa.2006.1712.

[27] Wang, C.M., Zhang, Y.Y., Ramesh, S.S., Kitipornchai, S. (2006). Buckling analysis of micro- and nano-rods/ tubes based on nonlocal Timoshenko beam theory. Journal of Physics D: Applied Physics, vol. 39, no. 17, p. 3904-3909, DOI:10.1088/0022-3727/39/17/029.

[28] Reddy, J.N. (2010). Nonlocal nonlinear formulations for bending of classical and shear deformation theories of beams and plates. International Journal of Engineering Science, vol. 48, no. 11, p. 1507-1518, DOI:10.1016/j.ijengsci.2010.09.020.

[29] Aydogdu, M. (2009). A general nonlocal beam theory: Its application to nanobeam bending, buckling and vibration. Physica E: Low-dimensional Systems and Nanostructures, vol. 41, no. 9, p. 1651-1655, DOI:10.1016/j.physe.2009.05.014.

[30] Thai, H.T. (2012). A nonlocal beam theory for bending, buckling, and vibration of nanobeams. International Journal of Engineering Science, vol. 52, p. 56-64, DOI:10.1016/j.ijengsci.2011.11.011.

[31] Gheshlaghi, B., Mirzaei, Y. (2012) Flexural sensitivity and resonance of cantilever micro-sensors based on nonlocal elasticity theory. Optics Communications, vol. 285, no. 12, p. 2798-2801, DOI:10.1016/j. optcom.2012.01.050.

[32] Civalek, Ö., Demir, Ç. (2011). Bending analysis of microtubules using nonlocal Euler-Bernoulli beam theory. Applied Mathematical Modelling, vol. 35, no. 5, p. 2053-2067, DOI:10.1016/j.apm.2010.11.004.

[33] Arash, B., Wang, Q. (2012). A review on the application of nonlocal elastic models in modeling of carbon nanotubes and graphines. Computational Materials Science, vol. 51, p. 303-313, DOI:10.1016/j. commatsci.2011.07.040.

[34] Reddy, J.N. (2007). Nonlocal theories for bending, buckling, and vibration of beams. International Journal of Engineering Science, vol. 45, no. 2-8, p. 288-307, DOI:10.1016/j.ijengsci.2007.04.004. 